\title{
Stationary parts of an EIT and Moreton wave: a topological model
}

\author{
C. Delannée ${ }^{1}$, J.-F. Hochedez ${ }^{1}$, and G. Aulanier ${ }^{2}$ \\ 1 Royal Observatory of Belgium, Brussels, Belgium \\ e-mail: ceaulanier@wanadoo.fr \\ ${ }^{2}$ LESIA, Meudon observatory, Meudon, France
}

Received 16 June 2006 / Accepted 30 August 2006

ABSTRACT

\begin{abstract}
Context. EIT and Moreton waves came into focus in 1997, when a propagating disturbance on a large area of the solar disc was discovered. The process generating the EIT and Moreton waves has been frequently discussed.

Aims. On May 2, 1998, a halo CME was observed related to an EIT wave, a Moreton wave, a X1 flare, radio emission sources, and dimmings. We studied this event to find the relation between all these structures.

Methods. We use and co-align multi-wavelength observations and the online potential field source surface (pfss) package.

Results. The observed EIT and Moreton waves present some brightenings that remain at the same location. We relate the connectivity of the coronal potential magnetic field to the stationary brightenings. We find that the areas where the magnetic field lines have drastic jumps of connectivity are cospatial to the stationary brightenings of the waves.

Conclusions. We conclude that the EIT and Moreton waves may be due to Joule heating resulting from the generation of electric currents in the neighboring area of the drastic jumps of magnetic connectivity, while the magnetic field lines are opening during a CME.
\end{abstract}

Key words. Sun: coronal mass ejections (CMEs) - Sun: magnetic fields - Sun: activity - Sun: flares - Sun: chromosphere Sun: corona

\section{Introduction}

Three wave-like structures with the length scale of the solar radius can be observed in three different spectral band passes. First, the Moreton waves are large arch-shaped brightenings propagating through the sun observed in $\mathrm{H} \alpha$ (Dodson 1949). They originate close to a flare that occurred few tens of seconds earlier (Warmuth et al. 2004a).

Second, the EIT waves are mainly observed with the Extreme ultra-violet Imaging Telescope (EIT, Delaboudinière et al. 1995) with the filter centered on $195 \AA$ and comprising the Fe XII emission line. Thompson et al. (1998) first reported an arch-shaped bright structure traveling across the sun that was named EIT wave. The morphologies and kinematics of this wave made the author suggest that it was very likely the Moreton wave. Thomspon et al. (1999) showed that the EIT and Moreton waves are quite cospatial when the two waves are observed in a same event.

Finally, X-ray waves were reported recently by Warmuth et al. (2005). X-ray waves also seem to originate from a flare, and are rather cospatial with EIT and Moreton waves. The fact that so many coronal and chromospheric waves are cospatial leads the authors to conclude that they are part of the same "decelerating disturbance".

All the waves reported in the articles mentioned above are each related to a flare, and seem to originate from them. So, the waves are interpreted as a magnetosonic wave initiated in a pressure pulse associated with the flare. Uchida (1968), Wu et al. (2001, 2005), and Wang et al. (2000) performed numerical simulations of magnetosonic waves initiated by a pressure pulse to reproduce certain properties of the observed wave-like structures. In this context, EIT waves could be used as proxies for coronal seismology, so as to derive the coronal physical parameters, which are important in terms of space weather applications (Ballai et al. 2005).

However, to our knowledge only four observed waves are almost full circles (the ones reported in Thompson 1998, 1999, 2000a). The circles of these four events are not complete: they present gaps of brightness. Apart from these rare cases, the waves are always observed with a quite narrow width: from 50 to 150 degrees in all studied spectral wavelengths (Warmuth et al. 2004a). A magnetosonic wave should almost always be a full circle even if the observed circle is dotted and deformed by the irregular medium in which it is propagating, as presented in the simulations (Wu et al. 2001, 2005; Wang et al. 2000). Moreover Delannée (2000) showed in four cases that some parts of the bright front of EIT waves can remain at the same location for $1 \mathrm{~h}$, which does not seem in accordance with a propagating wave.

Delannée \& Aulanier (1999) and Cliver et al. (2005) showed that nearly $100 \%$ of EIT waves are related to a CME. On the contrary, only $6 \%$ of flares are related to an EIT wave (Delannée \& Aulanier 1999), $1 \%$ of B-class flares are related to EIT waves (Cliver et al. 2005). Recently Chen (2006) claimed that none of 14 non-CME associated energetic flares are related to an EIT wave. Moreover the EIT waves are systematically associated in precise configuration with dimmed regions of the solar surface in $195 \AA$ (Delannée 2000). Harra \& Sterling (2001) showed that the dimmings occurring during a CME are due to density decrease and matter ejection. So, the EIT waves seem more related to CMEs than to flares. Therefore, EIT waves cannot be blast waves 
driven by a pressure pulse (Warmuth 2006). The Moreton waves are observed in red shift followed by blue shift in the wings of the $\mathrm{H} \alpha$ line (Warmuth et al. 2004b). The blue shift has a long lifetime so that it seems more related to matter ejection than to the oscillation of the matter in the wave. Thus, the Moreton wave is also closely associated with a CME. Since CMEs are strong drivers of space weather and since EIT waves appear to be an observational signature of their early development in the corona, understanding their physical properties has strong implications in terms of space weather predictions.

CMEs are believed to result from the fast expansion of previously stressed magnetic fields, driven by ideal or resistive MHD instabilities (see, e.g., the models of Amari et al. 1996; and Antiochos et al. 1999). When CMEs are launched, their expansion velocities quickly reach a significant fraction of the Alfvén speed. So CMEs lead to strong magnetic perturbations in the corona. The latter must naturally result in large-amplitude fastmode magnetosonic waves. While they propagate, such strong MHD waves can produce significant mass flows and pressure variations. These processes are clearly presented in 2.5D MHD calculations where the magnetic perturbation that is applied is modeled in the form of strong field line shearing motions (Wu et al. 1983). In the same context of CME related waves, Warmuth (2006) suggested a second possible process: EIT waves could be piston-driven waves as well as the CME shock fronts observed ahead of the CME as type II emission in radio wavelengths. In such cases, the expanding magnetic field would compress the plasma in the legs of the CME producing the bright EIT wave front. Based on the morphological properties of these waves observed in EUV in the low corona, Delannée \& Aulanier (1999) also conjectured that EIT waves could be due to the opening of magnetic field lines. However, it was shown that the EIT wave brightenings were stationary, and co-spatial with the footpoints of a separatrix surface, which is by definition a discontinuity in the field line connectivity. This first led to conjecture that the fast expansion of magnetic field lines, which should naturally compress the plasma at the boundaries between expanding and stable flux domains (so at least along separatrix surfaces) should therefore naturally lead to enhanced emission in coronal (EUV) lines.

This statement, though, is incomplete since stronger EUV emission can also be a result of plasma heating. It is well known that separatrix surfaces are natural locations for quasispontaneous current sheets (and therefore Joule heating) when they are perturbed by any motion (Low \& Wolfson 1988), and a fortiori by a CME-like expansion. Therefore, the association of EIT waves with separatrices is still possible in the context of Joule heating rather than plasma compression (Delannée 2000). Separatrices, though, appear not to be the only regions where narrow current layers can easily be generated (and dissipated). Quasi-separatrix layers, in which the magnetic field connectivity is continuous, but still has very sharp gradients over strongly squashed volumes, seem to have this physical property as well (see Démoulin 2005 and Aulanier et al. 2005). Actually, such drastic jumps of connectivity were successfully related to brightenings observed in many spectral lines, such as in EUV and in $\mathrm{H} \alpha$, as being due to magnetic reconnection in the corona (see Démoulin, 2005 and references therein). In this context, EUV emission may either be directly caused by early Joule heating, or by late chromospheric evaporation, while $\mathrm{H} \alpha$ brightenings probably result from the local heating of the chromosphere by non-thermal electrons accelerated high above in the corona from the reconnection regions (Schmieder et al. 1987).
Since the physical nature of EIT waves is still strongly debated, the objective of this paper was to test the relative strengths and weaknesses of each interpretation through the revisiting of a well documented event, already analyzed by several groups. We thus chose an event that occurred on May 2, 1998 and that comprised a X1 flare, an EIT wave, a Moreton wave, radio sources, and a halo CME. To our knowledge, 6 articles have already been published analyzing the relations of the different structures appearing during the event. Their results are summarized in Sect. 2. In the present study we re-analyze the same data, but using socalled "derotated base difference images" (DBDI). In these images, we then took special care to identify and study stationary brightenings that appear along the path of the EIT and Moreton waves. We overlaid the DBDI images on coronal field lines integrated from a potential field extrapolation. We found that the stationary brightenings are located in a region of drastic jumps in the magnetic field lines connectivity. This study first leads us to address the following question: observationally, are the stationary parts of the front of EIT waves a rare and peculiar class among all EIT wave related events (as advocated by Warmuth et al. 2004a) or are they generic and typical, so that the EIT waves really moving, that are detected with the EIT time cadence of image, are rather exceptional instead? We argue for the latter case. In this context, we suggest that the succession of stationary brightenings must result from the formation and dissipation of current sheets, progressively generated in large-scale quasiseparatrix layers, as the magnetic flux expands above the flare site and pushes up the overlaying transequatorial field lines.

\section{Summary of past studies}

Among the six papers that studied the May 2, 1998 event, five analyzed the observed waves. They all interpreted them as magnetosonic waves. The sixth paper addressed the issue of the large-scale dimmings developing in the corona during the whole event.

Thompson (2000b) showed that the EIT wave first appeared on the same image as the flare and as a large-scale dimming region on the solar disc, and that it was related to a halo CME. Warmuth et al. (2000) analyzed the structure and the velocity of the Moreton wave and its association with the flare. Pohjolainen et al. (2001), studied discrete radio bursts that occurred after the flare, which they not only related to the EIT and the Moreton waves, but also to fast disappearing transequatorial interconnecting loops (TIL) observed in SXR. They proposed that the blast wave produced the radio bursts along their path, as a result of some unknown instabilities in the large-scale coronal magnetic fields. Warmuth et al. (2004a) reinforced the relation between the EIT and the Moreton waves, suggesting that they are the coronal and the chromospheric part of the same wave. Warmuth et al. (2004b) integrated the light intensity over circles centered on the estimated Moreton wave starting point. The curves obtained of the mean intensity versus the distance to the starting point of the Moreton waves are called intensity profiles. The plot of the intensity profiles versus the radial distance from the starting point of the Moreton wave in the sequence of images gives the precise location of the wave front and the variation of its intensity as it propagates away from its origin. The wave front becomes fainter and its velocity smaller as it propagates. The facts that the wave front fits the arc of a circle, and is propagating lead the author to interpret it as a magnetosonic wave. Wang et al. (2002) extrapolated the coronal magnetic field in the potential field approximation over the whole corona and they showed how the large-scale magnetic field lines could be related 

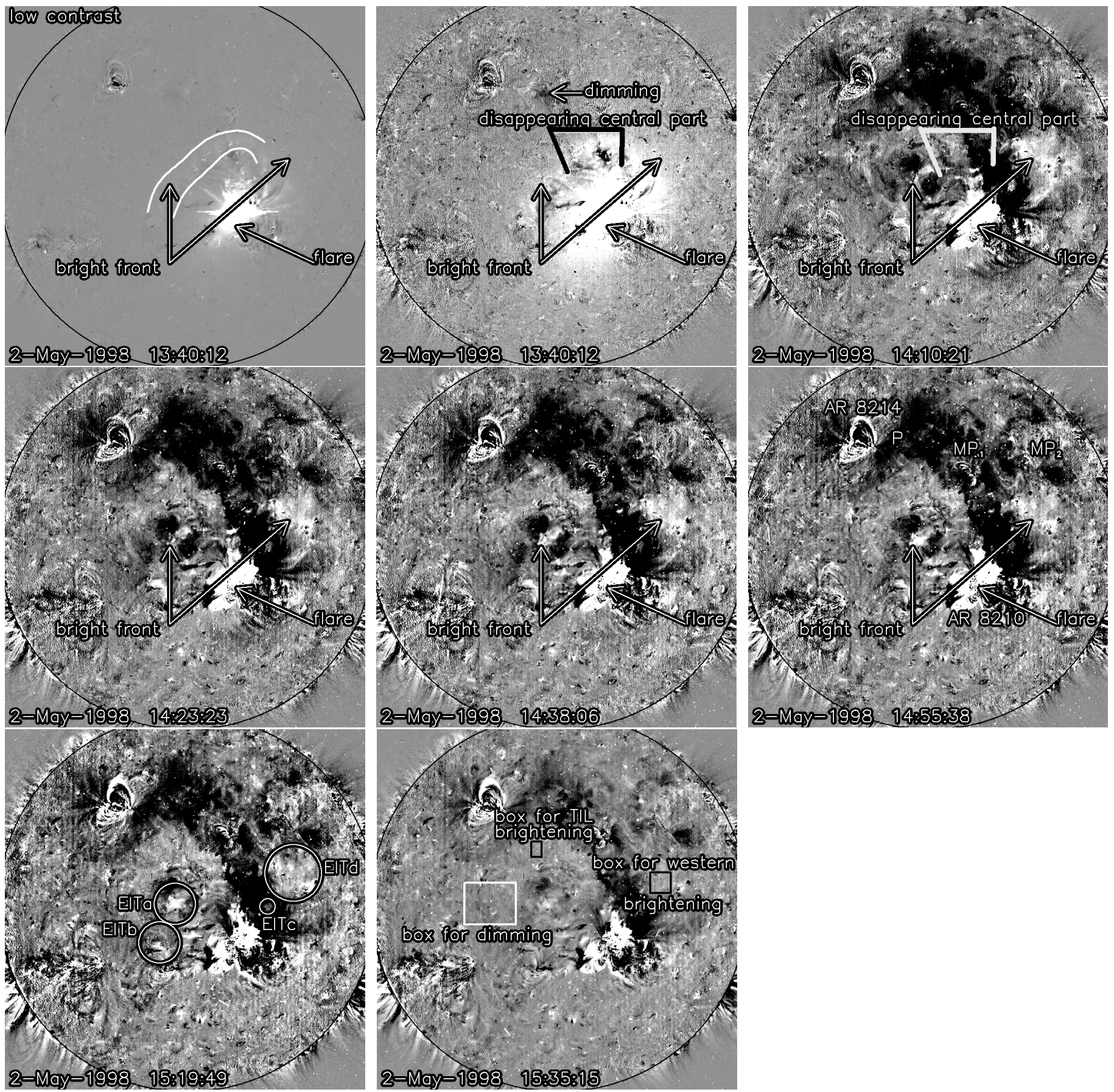

Fig. 1. SoHO/EIT observations using the $195 \AA$ filter on May 21998 from 13:40 UT to 15:35:15 UT. The images are differenced with a pre-event image at 13:19:46 UT. All images are corrected from differential rotation before subtraction. The first image is obtained using a low contrast, the following ones are obtained using a high contrast. The first and second images are obtained at the same time. Different structures are indicated by arrows, squares and circles. Over the squares were computed the mean light intensity presented in Fig. 2. The arrows are marked at the same location in all the images indicating the stationary brigthenings of the EIT wave.

to the dimmed regions, following the scenario first proposed in Delannée \& Aulanier (1999).

\section{Observations of the waves}

In this section, we analyze the EIT and Moreton waves produced during the event that occurred on May 2, 1998. The event begins with a X1 flare at 13:34:38 UT. Then a Moreton wave is detected in $\mathrm{H} \alpha$ and an EIT wave in $195 \AA$ a few minutes later. A halo CME appears in white light at 2 solar radii at 14:06 UT. We want to analyze the two observed waves, taking a special care to examine the stationary brightenings appearing on their passage.

\subsection{The EIT wave}

The images concerning the EIT wave are obtained with the $195 \AA$ filter of EIT. First, they are all corrected from the differential rotation, then their intensity is subtracted with a preevent image obtained at 13:19:46 UT to obtain derotated based difference images (DBDI). The DBDIs are presented in Fig. 1. 
EIT made synoptic images in four other wavelengths at that time, so the first image showing the EIT wave in $195 \AA$ is obtained at 13:40:12 UT. This image is also the first in $195 \AA$ showing the flare related to the EIT wave. There is a gap of $30 \mathrm{~min}$. between the two first images of the event. The following images were obtained with a 15 min cadence.

We calculated the time variation of mean brightness of the raw images in the three selected areas (see the image at 15:35:15 UT in Fig. 1) for 10 images recorded before and after the flare. The flare is about its maximum at 13:40:12 UT. Its brightness produces scattered light inside the telescope, which increases the mean intensity over the whole field of view. This scattered light induces the wide diffuse disc pointed as the flare on the second image at 13:40:12 UT in Fig. 1. The scattered light intensity decreases as the inverse square of the distance from the point on the CCD with the maximum intensity. Therefore, above the limb, the scattered light intensity is minimum. However, it is $8 \%$ higher than before the flare. This means that at least $8 \%$ of the computed mean intensities computed at 13:40:12 UT has to be taken into account when reading our figures, which do not incorporate this effect.

The EIT wave is the arch-shaped bright structure surrounding the northern edge of the flare (see the images at 13:40:12 UT in Fig. 1). It was also described by Warmuth et al. (2000, 2004a,b), Pohljolainen (2001), and Thompson (2000b). However, the bright front of the EIT wave has an arch shape in the DBDI at 13:40:12 UT only. In the following image at 14:10:21 UT, the central part of the arch has disappeared. Moreover, the eastern part and the western part of the arch stay at the same location as where they first appeared for a duration of $\approx 1.5 \mathrm{~h}$.

The variation of the mean light intensity in the box for the western part of the bright front shows that the intensity at this location stays $20 \%$ higher after the X1 flare than before it, for about $1 \mathrm{~h}$. Later, the intensity at this location begins to slowly decrease. Thus, the appearance of the stationary brightenings in $195 \AA$ can be found in the raw images and is not an artifact of the DBDI method.

Figure 1, E5 in Warmuth et al. (2004a), indicates the presence of an arch-shaped EIT wave front near the northern coronal hole at 14:10:21 UT. In the DBDIs, this latest front of the EIT wave does not appear (see north of the sun in Fig. 1 at 14:10:21 UT). This may be due to our differential rotation correction applied to the raw images to obtain the DBDIs. We tried to find this front in non-derotated running difference images or in the raw images. We found very faint brightenings at the edge of the coronal hole, which may be a signature of the EIT wave front. However, these brightenings are so faint and dispersed that we think that they are not related to each other and not to the EIT wave. Moreover, we could neither produce any image showing them, nor the mean intensity of a region to show that its brightness increases at 14:10:21 UT. However, Thompson (1998) showed in one other clear case that an EIT wave can produce brightenings at the edge of coronal holes.

Delannée (2000) proposed a general spatial relation between different structures appearing during an EIT wave (see the sketch in Fig. 10 in Delannée 2000). In the sketch summarizing the observations, we find, going away from the flare site: the flare, a deep dimming, an EIT wave stationary brightening, a faint dimming, and another deep dimming located at the footpoints of transequatorial loops connecting the flaring active region to another magnetic dipole located in the opposite hemisphere. Let us see if this sketch can be applied to this event.

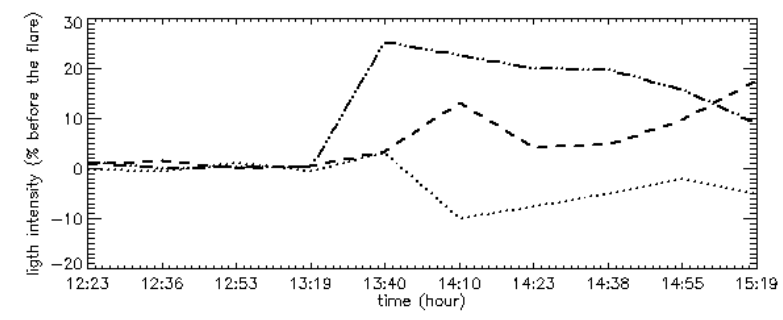

Fig. 2. Average percentage of the light intensity of the raw images over three selected area (shown as white and black rectangles in Fig. 1 at 15:35:15 UT) plotted against time. The dashed-dot-dot line is for the western brightening, the dashed line is for the TIL brightening, and the dotted line is for the dimming.

In the first image of the event, a dimming is observed near the active region AR 8210. Another one is located near the magnetic polarity (labeled $P$ on the image at 14:55:38 UT in Fig. 1) located in the opposite hemisphere. On the following images, the dimmings extend over a large area on the solar surface. We will analyze the western and the eastern stationary brightenings of the EIT wave separately as they are slightly different.

Firstly, on the east side, a faint dimming appears in the black box shown in Fig. 1 at 15:35:15 UT. This dimming is deepest just after the flare (see the mean light intensity plot in Fig. 2). Its intensity decreases by about $10 \%$. In this region the mean intensity increases back to normal at 14:55:38 UT, $\approx 1.5 \mathrm{~h}$ after the flare.

The Transequatorial Interconnecting Loop (TIL), mentioned in Pohljolainen (2001) and Wang (2002), is comprised in the white box of TIL brightening. In this region, the variation of the mean light intensity versus time shows that the loop does not disappear in $195 \AA$, but is rather associated with a light increase of about $15 \%$. The mean intensity in this region increases very slowly, from 14:10:21 UT to 15:19:49 UT. After 15:19:49 UT, the light intensity stays $15 \%$ higher than before the flare. The TIL disappearance in X-rays can be related to the CME: Hara et al. (2003) have indeed shown that large transequatorial loops can be illuminated in cool emission spectral lines, while above them other large transequatorial loops are observed opening. So, the disappearing TIL loop in X-rays may have been at higher altitude than the brightened TIL loop in $195 \AA$.

Therefore, the eastern part of the EIT wave almost follows the summary of observations produced in the sketch of Delannée (2000). In the direction away from the flare we see: the flare, a deep dimming, a stationary brightening of the EIT wave labeled EITa (see image at 15:19:49 UT in Fig. 1), a small deep dimming, a faint dimming, and finally a deep dimming located in the magnetic polarity $\mathrm{P}$ very close to the location where the TIL has been observed disappearing in soft-X rays. This succession of observed features away from the flare is summarized in a sketch in Fig. 3. The difference between the summary given by Delannée (2000) and this summary is the presence of a supplementary small deep dimming located between the stationary brightening and the faint dimming.

Secondly, in the west side, going from the flare site to the magnetic pore labeled $\mathrm{MP}_{2}$ (see the image at 15:19:49 UT in Fig. 1), we find a deep dimming close to the flare, then a small brightening labeled EITc (see Fig. 1), then another deep dimming, then a large stationary brightening of the EIT wave labeled EITd, and finally a deep dimming located near the magnetic pore labeled $\mathrm{MP}_{2}$. So, there is no faint dimming comprised between the stationary brightening of the EIT wave and the magnetic 


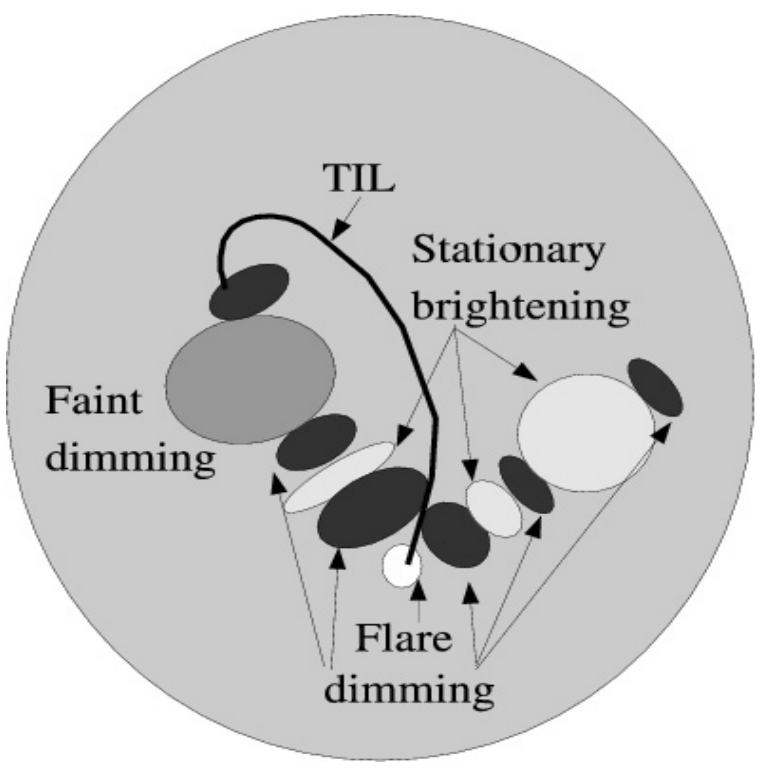

Fig. 3. Summary sketch of the eastern and the western stationary brightenings of the EIT wave. The observation shows different structures appearing in the following order when going away from the flare: the flare, a deep dimming, a stationary brightening of the EIT wave, and a deep dimming in a magnetic polarity located in the other hemisphere. The eastern stationary brightening of the EIT wave is related to a small deep dimming and a faint dimming located between the stationary brightening and the magnetic polarity P. The western stationary brightening does not show any faint dimming between the brightening and the magnetic polarity $\mathrm{MP}_{2}$.

pore, and there is no evidence of transequatorial loops connecting the magnetic pore to the active region. Moreover, the western stationary brightening is much larger (about $210 \pm 10 \mathrm{Mm}$ ) than the eastern one $(36 \pm 3 \mathrm{Mm})$. Again the summary of the observations described in Delannée (2000) can partially be applied to this stationary brightening of the EIT wave. The difference is the absence of a faint dimming between the stationary brightening of the EIT wave and $\mathrm{MP}_{2}$.

\subsection{The Moreton wave}

The data is obtained with the Patrol $\mathrm{H} \alpha$ Instrument at the Kanzelhöhe Solar Observatory. The time cadence of images is about $50 \mathrm{~s}$, but since the weather was cloudy above the observatory that day, some recorded images were very dark and not useful for our study. So there is a gap of data between 13:41:55 UT and 13:44:57 UT. The first image of the sequence showing the Moreton wave is at 13:38:57 UT. The flare at this time is well developed. Figure 4 presents $\mathrm{H} \alpha$ difference images with a preevent image at 13:35:54 UT. The two images obtained between 13:35:54 UT and 13:38:57 UT were too dark to be used. The differential rotation is not corrected because the time cadence is high enough to produce low artifacts, due to solar rotation, such as white and black vertical features in the difference images (see, for example, in active region AR 8214 in the image at 13:46:47 UT in Fig. 4).

The Moreton wave appears to be farther north than the flare location, just after a bright feature (Warmuth et al. 2000). The Moreton wave is arch-shaped. It is propagating from near AR 8210 to AR 8214 and to the two magnetic pores $\mathrm{MP}_{1}$ and $\mathrm{MP}_{2}$ located in the northern hemisphere. During its propagation the Moreton wave becomes fainter and thinner. The wave front consists of small brightenings (about $4 \mathrm{Mm}$ wide) and a diffuse arch. One brightening is illuminated before the diffuse front passes through it (see brightening $\mathrm{Hb}$ in the image at 13:46:47 UT in Fig. 4). It appears at 13:38:57 UT ahead of the bright front of the Moreton wave. The others are illuminated on the passage of the wave front (also described by Warmuth et al. 2004a). They can remain at the same place for 2 to $15 \mathrm{~min}$ before they faint. The closer they are to the flare, the longer they stay bright.

Warmuth et al. (2000) report that the velocity of the Moreton wave surprisingly increases between 13:40:51 UT and 13:44:57 UT. This is very different than the velocity plot versus time presented in Warmuth et al. (2004a) for the very same event in which the velocity monotonously decreases. So to clarify this issue, we computed the mean velocity between two different positions of a central point of the front going northward from the flare site (see Fig. 4). The velocity as a function of time is shown in Fig. 5. Our obtained velocities are among the lowest values reported in Fig. 8 E5 in Warmuth et al. (2004a). The velocity first decreases from about $473 \mathrm{~km} \mathrm{~s}^{-1}$ between 13:38:57 UT and 13:39:47 UT to about $237 \mathrm{~km} \mathrm{~s}^{-1}$ between 13:40:51 UT and 13:41:55 UT, then increases to about $376 \mathrm{~km} \mathrm{~s}^{-1}$ between 13:41:55 UT and 13:44:57 UT. The latest position of the Moreton wave is observed at 13:44:57 UT. The related brightenings stay at this same position at least until 13:46:47 UT. The following image that is sufficiently clear of cloud related darkenings for our analysis, is at 13:49:42 UT, i.e., about $5 \mathrm{~min}$. after the previous one. At this time, the stationary brightenings of the Moreton wave are no longer visible. So, they stay for two to five minutes at the same location. On the other hand, the diffuse front behaviours strangely after 13:44:57 UT: a western part of the diffuse front is going south-eastward (see the images at 13:44:57 UT, at 13:45:50 UT, and at 13:46:47 UT in Fig. 4). In the north, the diffuse front seems to stay at the same location between the brightenings labeled $\mathrm{Hf}$ and $\mathrm{Hg}$ in Fig. 4 at 13:46:47 UT. Between the brightenings labeled $\mathrm{Hg}$ and $\mathrm{Hh}$ in the same figure, the diffuse font of the Moreton wave goes slightly northward between the images at 13:44:57 UT and 13:46:47 UT. So, the average velocity of the Moreton wave becomes null between these two times. At 13:49:42 UT no part of the Moreton wave is visible any more.

Warmuth et al. (2004b) use an alternative method to study the Moreton wave front by integrating the light intensity over circles centered on the estimated Moreton wave starting point (see the plots in Fig. 2 E5 in Warmuth et al. 2004b). The obtained curves of the mean intensity versus the distance to the starting point of the Moreton waves are named intensity profiles. Plotting the intensity profiles obtained in the sequence of images gives the precise location of the wave front and the variation of its intensity as it propagates away from its origin. Using this method Warmuth et al. (2004b) showed that the wave front becomes fainter as it propagates. We reanalyze those intensity profile plots. The drawn curves show several pics of intensity. Those pics remain at the same distance from the origin of the wave as the time goes. This is coherent with the presence of the stationary brightenings that we pointed in Fig. 4.

\subsection{Spatial relation between the EIT and Moreton waves}

To have some more insight into the relation between the EIT wave and the Moreton wave, we overlaid their fronts on the EIT images (Fig. 6). The contours are drawn at $3 \%$ of the maximum intensity of the $\mathrm{H} \alpha$ image. The contours of the Moreton wave are obtained by selecting a large region around the wave front. At the chosen contours of intensity level, the flare is a very large 

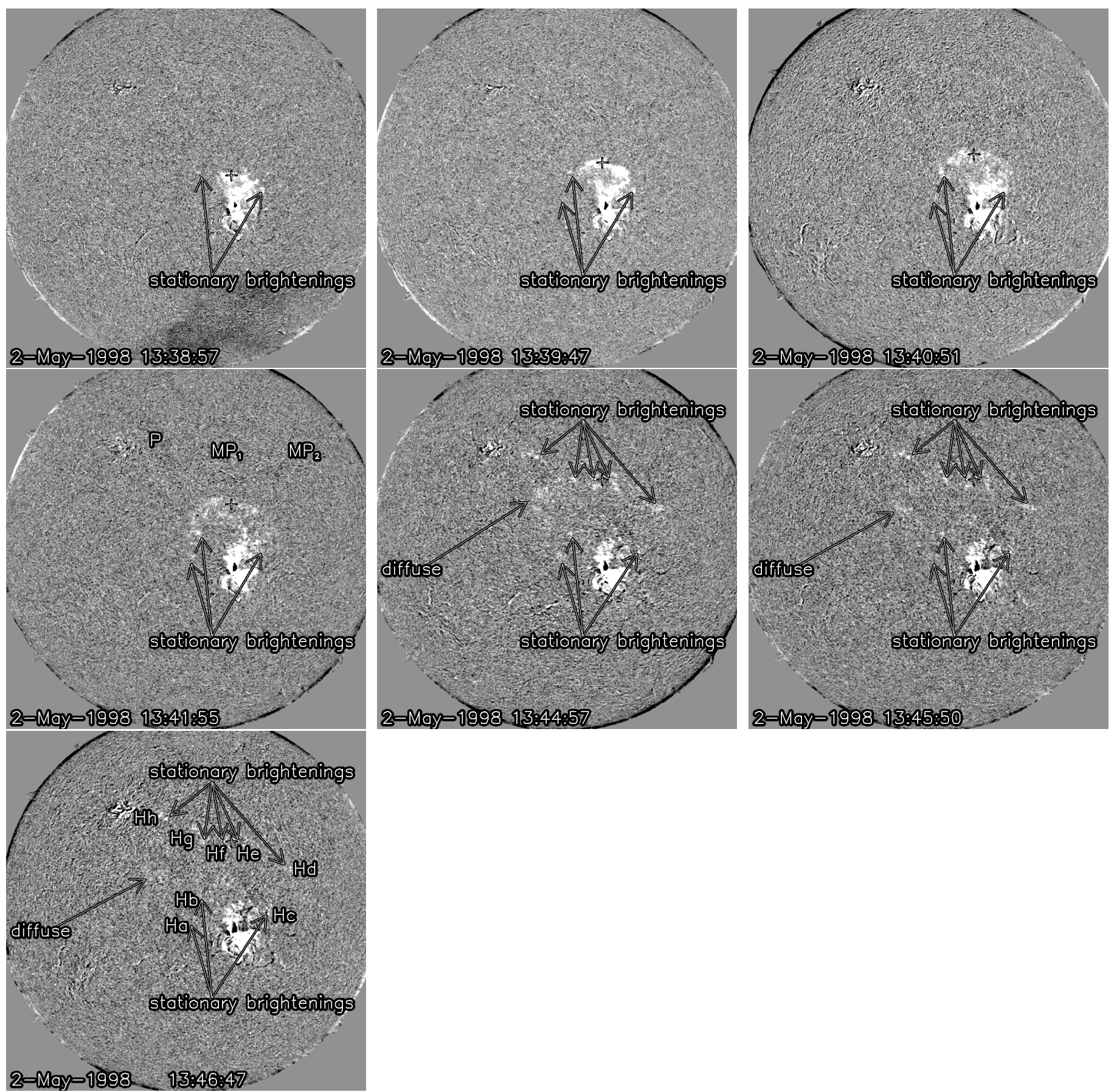

Fig. 4. Kanzelhöhe Solar Observatory observations in $\mathrm{H} \alpha$ on May 21998 from 13:38:57 UT to 13:46:57 UT. The images are based difference images of the Moreton wave with a pre-event image at 13:35:54 UT. The arrows are drawn at the same locations in all the images. The Labels MP ${ }_{1}$ and $\mathrm{MP}_{2}$ are written at the same location as in Fig. 1 at 14:55:38 UT.

feature. So, to focus on the Moreton wave front we removed the flare site from the selected region.

The Moreton wave front at 13:40:51 UT and EIT wave front at 13:40:12 UT are not fully cospatial (see left image in Fig. 6). Although the $\mathrm{H} \alpha$ contours are obtained 39 s later than the EIT image, the Moreton wave front is comprised inside the EIT wave front. The following image in $195 \AA$ is at 14:10:21 UT. As the Moreton wave front stays at the same place until it faints, we overlaid the Moreton wave front at 13:44:57 UT and the EIT image at 14:10:21 UT (see the right image in Fig. 6). The brightenings $\mathrm{Ha}$ and $\mathrm{Hb}$ do not correspond to the stationary brightening EITa: they are in the coronal dimming between the flare site and the brightening EITa and at the border of EITa. The H $\alpha$ stationary brightenings labeled $\mathrm{He}, \mathrm{Hf}, \mathrm{Hg}$, and $\mathrm{Hh}$ are also in the dimmings observed in $195 \AA$ and located in the magnetic pores $\mathrm{MP}_{1}$, $\mathrm{MP}_{2}$, and $\mathrm{P}$. While those 6 stationary $\mathrm{H} \alpha$ brightenings are in coronal dimmings, 2 others are in coronal brightenings. Hc and $\mathrm{Hd}$ are where the coronal stationary brightenings EITc and EITd are. The diffuse bright front of the Moreton wave in the images at 13:44:57 UT, at 13:45:50 UT, and at 13:46:47 UT (see Fig. 4) is comprised in the eastern brightening in the black box drawn on the image at 15:15:35 UT in Fig. 1. So, it seems very difficult to understand the physical relation between the stationary brightenings of the Moreton and EIT waves with this overlay only. 


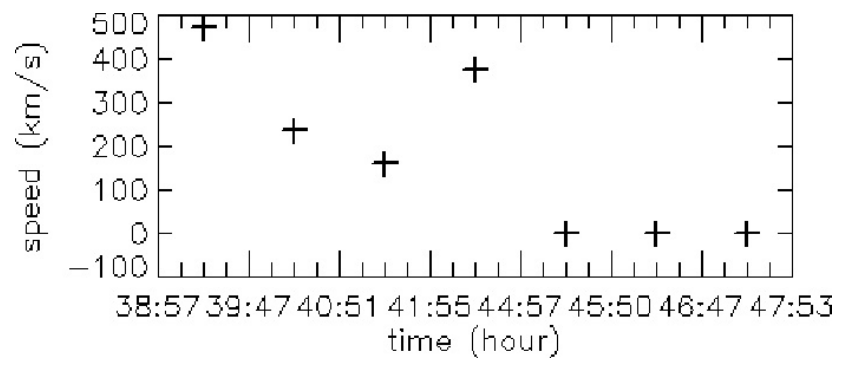

Fig. 5. Mean velocity between two images of a central point of the Moreton wave front shown by the crosses drawn on the images in Fig. 4).

\section{Magnetic field topology corresponding to the stationary brightenings of the EIT and Moreton waves}

We showed that stationary brightenings are created on the passage, and in one case before the passage of the EIT and Moreton waves. Démoulin (2005) reviewed the many spatial relations between brightenings observed in many emission lines and the topology of the magnetic field in the corona. Those brightenings are always embedded in the drastic jump of connectivity of magnetic field lines that are preferable places for the generation of electric currents and therefore Joule heating. So, we want to find the spatial relation between the EIT and Moreton waves stationary brightenings, and the magnetic field lines. We used the potential field source surface (pfss) package of the solar software, and retrieved from URL www.lmsal.com/solar/soft/psff_links/ the file bfield_19980502_12_04_00.sav containing the results of the simulation of the magnetic field performed by Schrijver \& deRosa (2003). The simulation is done in spherical coordinates. On one boundary a synoptic map of the magnetic field data, obtained by the Michelson Doppler Instrument (MDI) on board SoHO, is applied. On the other boundary, the opening of the coronal magnetic field is obtained by sources placed randomly so as to obtain the better mapping of the corona morphology at the time of the observation. A model of migration, rotation, reconnexion, and emergence of magnetic fluxes is applied to compute the potential magnetic field above the photosphere. From this calculation, we chose to draw the magnetic field lines that have one or two footpoints in the EIT and $\mathrm{H} \alpha$ stationary brightenings and the ones that are overlying them. We want to point out the magnetic field line connectivity lying in the EIT and Moreton stationary brightenings. The results retrieved from Schrijver and deRosa do not allow us to compute the exact location of a separatrix or a quasi-separatrix layer. So, we cannot argue about the exact topology of the brightened regions, but we can determine if a jump of connectivity exists or not. We present the results of this analysis in Fig. 7. The left image is the magnetogram with the magnetic field lines chosen to represent their connectivity in the stationary brightenings in $195 \AA$ and in $\mathrm{H} \alpha$. The middle image in Fig. 7 is the EIT image at 14:10:21 UT with some magnetic field lines. The right image in Fig. 7 is the $\mathrm{H} \alpha$ image at 13:44:57 UT with the white contours of the stationary brightenings, the light grey contours of the positive magnetic polarities, the dark grey contours of the negative magnetic polarities, and some magnetic field lines.

Here we describe the magnetic field line connectivity embedded in the stationary brightenings of the EIT wave. There are two different topologies in the eastern brightening. The brightening in the circle labeled EITa in Fig. 1 at 15:19:49 UT, lies in a drastic jump of connectivity of the magnetic field lines. The red magnetic field lines connect AR 8210 to the border of the region in the circle EITa. All the blue magnetic field lines connect the border of the region in the circle EITa to a small magnetic polarity lying approximately in the center of the circle EITa. Then, some magnetic field lines drawn in pink connect the opposite border of the circle EITa to the magnetic polarity P. All these lines are overlaid by large-scale green magnetic field lines connecting the active region 8210 and to the magnetic polarity $\mathrm{P}$ located in the opposite hemisphere. So, the general magnetic field topology of this region resembles a quadrupolar topology. More in the south, the magnetic field is open (see the white magnetic field line drawn in the left and middle images in Fig. 7, also described by Wang et al. 2002). The open magnetic field lines define a separatrix in the magnetic field topology where the magnetic field connectivity jumps from the sun to the solar wind. The brightening in the circle labeled EITb in Fig. 1 at 15:19:49 UT is in the region of open magnetic field lines.

The magnetic field structure of the eastern stationary brightening of the EIT wave is divided into two parts corresponding to the brightenings labeled EITc and EITd in the image at 15:19:49 UT in Fig. 1. There is a first small brightening EITc close to the flare embedded in the jump of connectivity between the yellow field lines and the small cyan field lines, overlaid by the red field lines. Going west-northward lies a dimming. There all the field lines have one footpoint in the dimmed region and the other footpoint in the flaring active region AR 8210. There is no jump of connectivity of the magnetic field in this region at the length scale of the region. Then we find another jump of connectivity between the red and the pink field lines, overlaid by the large-scale green field lines. The stationary brightening EITd is embedded in the blue and the pink field lines.

No magnetic field line links AR 8210 to the disappearing central part of the EIT wave between the western and the eastern stationary brightenings of the EIT wave. In this part all the magnetic field lines coming from AR 8210 go directly to the magnetic pore $\mathrm{MP}_{1}$ located in the northern hemisphere. Under those lines, the magnetic field line connectivity jumps from one small magnetic polarity to another.

The overlay of the magnetic field lines and the $\mathrm{H} \alpha$ stationary brightenings show that all of them are lying in magnetic polarities where the magnetic field lines locally converge. For the brightenings labeled $\mathrm{Ha}, \mathrm{Hb}, \mathrm{Hc}, \mathrm{He}$, and $\mathrm{Hh}$ in Fig. 4 at 13:46:47 UT, the magnetic field lines (drawn in red, cyan, yellow, and green, respectively) are directly connected to AR 8210 . For the brightenings labeled $\mathrm{Hd}, \mathrm{Hf}$, and $\mathrm{Hg}$ (drawn in pink, dark pink, and violet, respectively) all the magnetic field lines have no footpoint in the active region AR 8210, but have them in the neighboring $\mathrm{H} \alpha$ brightening instead. All the brightenings lie at the magnetic field lines'jump of connectivity. $\mathrm{Ha}$ and $\mathrm{Hb}$ lie at the edge of the same jump of connectivity as EITa, at the footpoint of red magnetic field lines that have their other footpoint in the flaring region. Hc lies at the edge of the same jump of connectivity as EITc, at the footpoint of the yellow magnetic field lines that have their other footpoint in the flaring region. Hd lies in the jump of connectivity drawn by the blue and the pink magnetic field lines. He lies at the jump of connectivity drawn by the green and the dark pink magnetic field lines; the green magnetic field lines have their other footpoint in the flaring region. Hf lies at the jump of connectivity drawn by the dark pink and the violet magnetic field lines. 



Fig. 6. Overlay of the EIT wave front and the Moreton wave front: in the right image at 13:41 UT in EIT and 13:40:51 UT in $\mathrm{H} \alpha$, and in the left image at 14:11 UT in EIT and
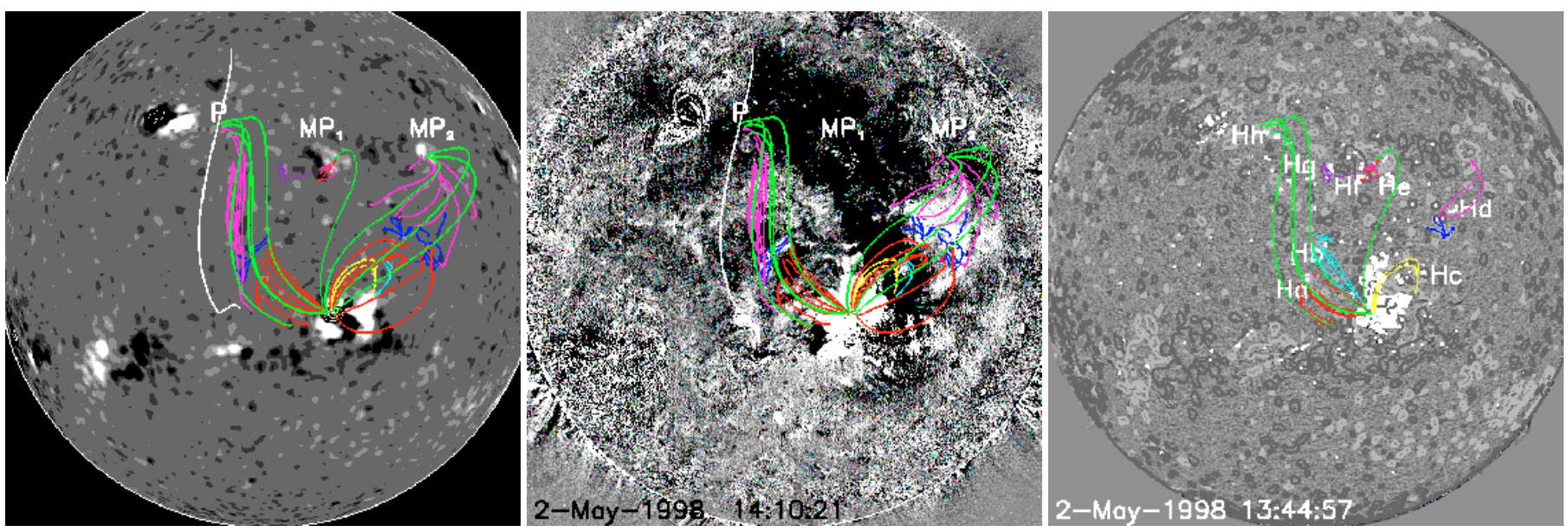

Fig. 7. Left image: magnetogram and the extrapolated magnetic field lines obtained by Schrijver \& deRosa (2003). The magnetic field lines at the border of regions of different connectivity are drawn in different colours. The white magnetic field lines are opened. Middle image: overlay of the EIT image at 14:10:21 UT and some magnetic field lines. Right image: overlay of the H $\alpha$ image at 13:44:57 UT and some magnetic field lines.

\section{Discussion}

This analysis shows that the EIT and Moreton waves produced on May 2, 1998, are certainly different parts of the same wave like-structure because they are almost cospatial. However, the Moreton wave bright front is comprised in the EIT wave bright front. The Moreton wave bright front consists of a moving diffuse arch and stationary brightenings. The EIT image cadence is too poor to see an EIT wave diffuse arch moving, only the central disappearing part of the diffuse arch is observed. However, the 1 min cadence of images of the Transition Region and Coronal Explorer (TRACE) allowed us to observe a moving EIT wave diffuse arch on June 13, 1998 (Delannée 2000). So, we suggest here that with a better time cadence of image the EIT wave diffuse arch produced on May 2, 1998, should also be observed moving in the same manner as the Moreton wave diffuse arch. The three last available images of the Moreton wave show that its bright front is almost stationary.

The EIT and Moreton waves both consist of stationary brightenings produced most of the time on their passage. However, one stationary brightening is produced before the Moreton wave passes by its location. The EIT and $\mathrm{H} \alpha$ stationary brightenings are all related to the topology of the magnetic field. They are all produced in places of drastic magnetic field line jumps of connectivity.

The question resulting from all these facts is: how may we understand these EIT and Moreton waves? The EIT stationary brightenings are produced in magnetic field line connectivity jumps. In these locations, the opening of the magnetic field lines would produce electric currents. These electric currents may dissipate their energy in the plasma by Joule heating. Therefore, in these jumps of connectivity the plasma would be brightened.

However, we remark that the eastern brightening EITa is much thinner than the western brightening EITd. This may be due to some subsequent reconnexion that certainly took place near the magnetic polarity $\mathrm{P}$. These subsequent reconnexions may have lead to the expansion of the pink magnetic field line connecting EITa to P (see middle image of Fig. 7). This expansion may have produced matter depletion at their footpoints and therefore the deep dimming near EITa. Even if the pink magnetic field lines may have been the places of generation of electric currents, the Joule heating in the depleted coronal plasma may have not been efficient enough to brighten it in the Fe XII emission line, which is comprised in the $195 \AA$ filter band pass (see left image in Fig. 8).

On the other hand, the western brightening EITd is very large, which may be due to the absence of expansion of the pink magnetic field lines connecting the border of EITd to the magnetic pore $\mathrm{MP}_{2}$ (see middle image of Fig. 7). Therefore, the coronal plasma lying in these pink magnetic field lines may have been brightened by Joule heating due to the generation of electric currents along the pink magnetic field lines while the overlying green magnetic field lines are opening (see right 

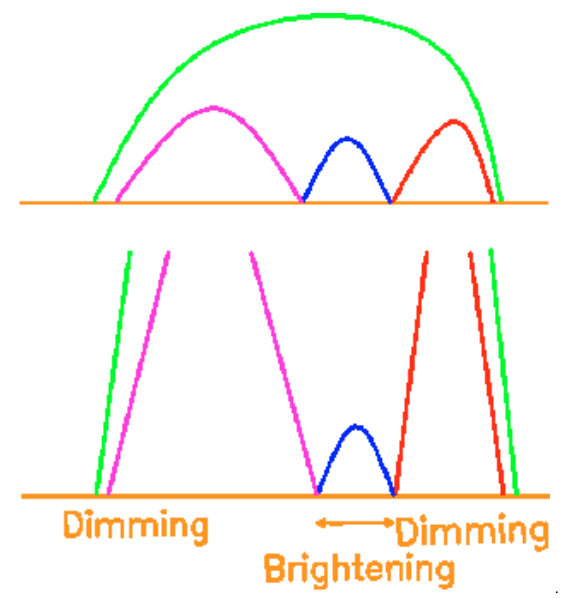
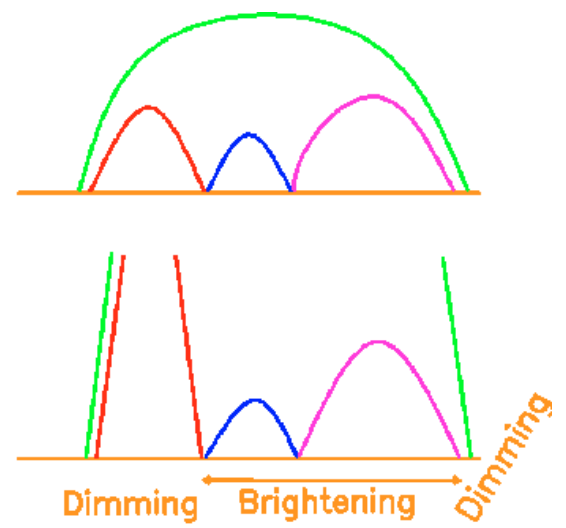

Fig. 8. Sketches showing the expansion of the magnetic field line that may be responsible for the EITa (left image) and EITd (right image) stationary brightenings of the EIT wave. image in Fig. 8). The sketch presented in Delannée (2000) shows that a faint dimming should exist between the stationary EITd and the magnetic pore $\mathrm{MP}_{2}$. This faint dimming should appear if the opening of the green magnetic field lines lead to plasma depletion at a typical temperature of the emission of the Fe XII spectral line. Therefore, the altitude where the plasma depletion took place was not at the required temperature to be observable in Fe XII. This dimming was maybe observable in another spectral emission line that day.

The $\mathrm{H} \alpha$ brightenings are very small compared to the EIT ones, which means that the processes of the $\mathrm{H} \alpha$ emission are quite different than the ones of the Fe XII emission. The $\mathrm{H} \alpha$ brightenings are produced in magnetic field polarities where the connectivity of the magnetic field lines jumps. In the reconnection site, electrons are accelerated. They follow the magnetic field lines to impact the chromosphere and produce the $\mathrm{H} \alpha$ brightenings. This process may explain the appearance of the stationary brightenings $\mathrm{Ha}, \mathrm{Hb}, \mathrm{Hc}, \mathrm{He}$, and $\mathrm{Hh}$ that are at one footpoint of magnetic field lines, while their other footpoint is in the flaring region. The brightenings $\mathrm{Hd}$, $\mathrm{Hf}$, and $\mathrm{Hg}$ are in magnetic polarities where the connectivity of the magnetic field lines jumps from one magnetic polarity to the following one. Non-thermal electrons can jump from one magnetic field line to another one. So, they may follow the magnetic field lines and create a $\mathrm{H} \alpha$ brightening in the neighboring magnetic polarity. More precisely, the accelerated electrons may have followed the green magnetic field line from the flare site to $\mathrm{He}$, then they may have followed the pink magnetic field lines from He to Hf, and the violet magnetic field lines from $\mathrm{Hf}$ to $\mathrm{Hg}$. Hd may have been created by the electric currents generated between the blue and green magnetic field lines, which define a jump of connectivity of the magnetic field lines. So, the stationary brightenings in EIT and in $\mathrm{H} \alpha$ are much more like sympathetic flares (as mentioned by Podladchikova \& Berghmans 2006), even if the EIT stationary brightenings are quite larger than the $\mathrm{H} \alpha$ brightenings.

As $\mathrm{Ha}, \mathrm{Hb}, \mathrm{He}$, and $\mathrm{Hh}$ are at the footpoint of magnetic field lines connecting the flaring regions, which are opening, they are lying in coronal dimmings. $\mathrm{Hf}$ and $\mathrm{Hg}$ are not at the footpoint of opening magnetic field lines. However, they are lying in a region overlaid by large-scale magnetic field lines connecting the flaring region. So, they are also lying under a depleted corona. $\mathrm{Hd}$ lies at the footpoint of the blue closed magnetic field lines overlaid by the green opening magnetic field lines, so this $\mathrm{H} \alpha$ brightening is lying in an EIT stationary brightening. Finally, the presence of Hc at the limit between EITc and a dimming is certainly due to the opening of the yellow magnetic field lines. However in this case Hc should be lying in the dimming. This discrepancy is certainly due to the many approximations made in this study. Therefore, the EIT and Moreton wave stationary brightenings are spatially related to the observed dimmings as they seem to be produced by the opening of magnetic field lines occurring during a CME.

Most of the time, the EIT and Moreton wave stationary brightenings appear as the moving diffuse arches of the waves are passing through the brightened locations. This study just gives some conjectures of the EIT and Moreton waves'stationary brightenings together with the magnetic field topology, but do not prove the model given above. So, an ambiguity remains: are they produced by the opening of magnetic field lines or are they produced by the perturbation of the ambient coronal magnetic field while a magnetosonic wave passes through them? We can resolve this ambiguousness if we resolve the following question: how is the diffuse moving arch of the EIT and Moreton wave generated?

The shape and the propagation of the diffuse moving arch very much resembles a wave that is driven by the flare related plasma heating. However, such pressure-pulse models cannot explain several aspects of the EIT and Moreton waves. First, their shapes are neither full circles nor continuous all around the flare site. Second, the starting point of the Moreton wave is not exactly located at the flare site: there is at least $25 \mathrm{Mm}$ between the starting point and the center of the $\mathrm{H} \alpha$ flare (Warmuth et al. 2004a). Moreover, the estimated instant of the initiation of the Moreton wave is at least one minute later than the maximum of the flare (Warmuth et al. 2004a). Finally, the majority of flares do not produce any observable waves.

On the contrary, the EIT waves are systematically associated with large dimming areas of the solar surface and a CME just as the Moreton wave propagating front is systematically followed by ejection of matter. In this context, the observed moving fronts could be modeled by fast-mode magnetosonic waves, where the CME acts as a magnetic pulse. But we argue that observations do not support this interpretation as well. A priori, the dimmings could be consistent with the rarefaction that is found in some fast-mode magnetosonic wave models (Wu et al. 1983), but their observed persistance during several hours (far longer than the Alfvén time scales) is inconsistent with the short relaxation time of the plasma that is expected after a wave has passed through it. The existence of dimmings is therefore not a supportive argument for a magnetic pulse wave interpretation. So, we believe that the diffuse moving arches of the EIT and Moreton waves are also consequences of the restructuration of the coronal magnetic field during a CME. 
The diffuse moving arch could be due to the generation of electric current in the moving layers formed at the place where rapidly expanding magnetic field lines encounter the surrounding not yet expanding magnetic field lines. The magnetic field lines originating from a magnetic polarity connect all the surrounding magnetic polarity almost forming a circle. As the eruption takes place in a narrow portion of the arch, the generated electric currents would be arch shaped and very rarely a full circle. It would propagate away from the flare site as the magnetic field lines are opening. The velocity of propagation would decrease as the magnetic field lines become more radial. The brightness would decrease as the magnetic field lines become higher and the generated electric currents heat less dense plasma. Finally, the generated brightness would stop at the boundary of the solar surface region inside which the magnetic field lines are opening, i.e. in layers of magnetic field lines'jumps of connectivity. The fact that the Moreton wave bright front is inside the EIT wave bright front may be due to 3D geometry of the generated electric currents during the restructuration of the magnetic field.

\section{Conclusion}

We studied an event produced on May 2, 1998. This event consisted of a flare located near AR 8210, of a halo CME, of dimmings on the solar surface, of a Moreton wave and of an EIT wave. This event was already studied in 5 articles that concluded that the EIT and Moreton waves are probably magnetosonic waves. We found that the EIT and Moreton waves present some stationary brightenings remaining at the same location for several minutes. So, we here address the remark to the solar community that using another process of study, one may find some stationarity in a large number of EIT and Moreton waves.

We related the stationary brightenings to the magnetic field topology. The brightenings in both spectral observations are embedded in the drastic jumps of connectivity of the magnetic field. They could be generated by Joule heating or electron acceleration occurring while the magnetic field lines are opening during the CME. However, the brightness and the exact shape and thickness of the brightenings result in competitive processes: Joule heating versus density decrease. If the model of the opening of the magnetic field can predict the major part of the dimmed region observed on the solar surface and the location of some stationary structures appearing on the passages of the EIT and Moreton waves, it could not predict their exact appearance unless we had a full numerical simulation of the event.

A diffuse arch observed in $\mathrm{H} \alpha$ is moving, which could not be related to any jumps of connectivity of the magnetic field. We speculate that a moving diffuse arch could also be observed in EIT if its image time cadence had been better. The process leading to the appearance of the diffuse moving arch of the EIT and Moreton waves is still not proved with this study. However, the fact that they are observed closely related to a CME leads us to suggest that they also result from the restructuration of the coronal magnetic field occurring during the CME. Further works are needed to analytically and numerically simulate the creation of these waves with a model of the opening of magnetic field lines.

Acknowledgements. We thank Wolfgang Otruba for providing us with the $\mathrm{H} \alpha$ data and Brigitte Schmieder for fruitful discussions.

\section{References}

Amari, T., Luciani, J.-F., Aly, J.-J., \& Tager, M. 1996, ApJ, 306, 913 Antiochos, S. K., De Vore, C. R., \& Klimtchuk, J. A. 1999, ApJ, 510, 485 Aulanier, G., Pariat, E., \& Démoulin, P. 2005, A\&A, 444, 961

Ballai, I., Erdélyi, R., \& Pintér, B. 2005, ApJ, 633, L145

Chen, P. F. 2006, ApJ, 641, L153

Cliver, E. W., Laurenza, M., Storini, M., \& Thompson, B. J. 2005, ApJ, 631, 604 Delaboudinière, J.-P., Artzner, G. E., Brunaud, J., et al. 1995, Sol. Phys., 162, 291

Delannée, C. 2000, ApJ, 545, 512

Delannée, C., \& Aulanier, G. 1999, Sol. Phys., 190, 107

Delannée, C., Delaboudinière, J.-P., \& Lamy, P. 2000, A\&A, 355, 725

Démoulin, P. 2005, Adv. Space Res., 37, 1269

Dodson, H. W. 1949, ApJ, 110, 382

Handy, B. N., Acton, L. W., Kankelborg, C. C., et al., 1999, Sol. Phys., 187, 229 Harra, L., Matthews, S. A., \& van Driel-Gesztelyi, L. 2003, ApJ, 598, L59

Harra, L., \& Sterling, A. C. 2001, ApJ, 561, L215

Khan, J. I., \& Aurass, H. 2002, A\&A, 383, 1018

Low, B. C., \& Wolfson, R. 1988, ApJ, 324, 574

Podladchikova, O., \& Berghmans, D. 2006, A\&A, in press

Pohjolainen, S., Maia, D., Pick, M., et al. 2001, ApJ, 556, 421

Schmieder, B., Malherbe, J. M., Simnett, G. M., Forbes, T. G., \& Tandberge-Hanssen, E. 1990, ApJ, 356, 720

Schrijver, C. J., \& Derosa, M. L. 2003, Sol. Phys., 212, 165

Shiota, D., Isobe, H., Chen, P. F., et al. 2005, ApJ, 634, 663

Thompson, B. J., Plunkett, S. P., Gurman, J. B., et al. 1998, Geophys. Res., Lett., 25,2461

Thompson, B. J., Gurman, B. J., Neupert, W. M., et al. 1999, ApJ, 517, L151

Thompson, B. J., Reynolds, B., Aurass, H., et al. 2000a, Sol. Phys., 193, 161

Thompson, B. J., Cliver, E. W., Nitta, N., Delannée, C., \& Delaboudinière, J.-P. 2000b, Geophys. Res. Lett., 27, 1431

Uchida, Y. 1968, Sol. Phys., 4, 30

Wang, T., Yan, Y., Wang, J., Kurokawa, H., \& Shibata, K. 2002, ApJ, 572, 580

Wang, Y. M. 2000, ApJ, 543, L89

Warmuth, A. 2006, in The high energy solar corona : waves, eruptions, particles, ed. K. L. Klein, \& A. MacKinnon (Springer), Lecture Notes in Physics, in press

Warmuth, A., Hanslmeier, A., Messerotti, M., et al. 2000, Sol. Phys., 194, 103

Warmuth, A., Vrŝnak, B, Magdalenić, J., Hanslmeier, A., \& Otruba, W. 2004a, A\&A, 418, 1101

Warmuth, A., Vrŝnak, B, Magdalenić, J., Hanslmeier, A., \& Otruba, W. 2004b, A\&A, 418, 1117

Warmtuh, A., Mann, G., \& Aurass, H. 2005, ApJ, 626, L121

Wu, S. T., Hu, Y. Q., Nakagawa, Y., \& Tandberg-Hanssen, E. 1983, ApJ, 266, 866

Wu, S. T., Zheng, H., Wang, S., et al. 2001, JGR, 106, 25089

Wu, S. T., Li, B., Wang, S., \& Zheng, H. 2005, JGR, 100, A11102 